\title{
Primer registro de un posible híbrido homoploide y de un tetraploide en el género Chaetanthera Ruiz \& Pav. (Asteraceae) en Chile
}

\section{First report of a possible homoploid hybrid and a tetraploid in the genus Chaetanthera Ruiz \& Pav. (Asteraceae) in Chile}

\author{
Carlos Baeza Perri ${ }^{1}$, Eduardo Ruiz ${ }^{1} \&$ Jaime Espejo $^{2}$ \\ ${ }^{1}$ Departamento de Botánica, Facultad de Ciencias Naturales y Oceanográficas, Universidad de Concepción, Casilla 160-C, \\ Concepción, Chile. \\ 2Programa de Postgrado, Facultad de Ciencias Forestales, Universidad de Concepción, Victoria 631, Casilla 160-C, \\ Concepción, Chile \\ cbaeza@udec.cl
}

\section{RESUMEN}

En este trabajo se reportan cariotipos detallados de C. elegans Phil., C. x serrata Ruiz \& Pav. y C. chilensis (Willd.) DC. Además, se registra por primera vez la presencia de un posible híbrido homoploide entre C. chilensis y C. elegans.y de un tetraploide en Chaetanthera elegans.

Palabra clave: Chaetanthera $x$ serrata, híbrido homoploide, poliploidía, Chile.

\begin{abstract}
Detailed karyotypes of C. elegans Phil., C. x serrata Ruiz \& Pav. and C. chilensis (Willd.) DC. are given. In addition, the result of the present contribution are the first report of a possible homoploid hybrid between C. chilensis and C. elegans, and a tetraploid in Chaetanthera elegans.
\end{abstract}

KEYwords: Chaetanthera x serrata, homoploid hybrid, polyploidy, Chile.

\section{INTRODUCCIÓN}

Chaetanthera Ruiz \& Pav. (Asteraceae) es un género endémico de América del Sur y está compuesto por 30 especies, una variedad y dos híbridos, constituyéndose en uno de los géneros más numerosos de la tribu Mutisieae en Sudamérica (Davies 2010). En Chile se encuentran creciendo la mayoría de las especies. Cabrera (1937) considera a Chaetanthera formado por 42 especies, puesto que incluye en este género a especies de Oriastrum Poepp. \& Endl. que en la actualidad es reconocido como un género válido, que habita fundamentalmente en la parte alta de la Cordillera de los Andes de Bolivia, Perú, Argentina y Chile (Davies 2010). Cabrera (1937) divide al género en 7 subgéneros, principalmente sobre la base del hábito y de la morfología de las brácteas del involucro. Uno de estos subgéneros lo constituye Proselia, con las especies: $C$. serrata, $C$. elegans, C. brachylepis, C. chilensis y C. valdiviana. En la actualidad no se reconoce el subgénero Proselia y sólo se consideran válidas las especies $C$. chilensis, $C$. elegans y C. serrata, ahora pertenecientes al subgénero Chaetanthera.
Además, esta última especie es considerada un híbrido entre las dos primeras. (Davies 2010). Hershkovitz et al. (2006), consideran a estas tres especies estrechamente emparentadas, lo que sería congruente con la relación genealógica propuesta por Davies (2010). Si esta hipótesis es correcta, entonces C. $x$ serrata debería constituir un alopoliploide, dado que es lo más probable encontrar en híbridos interespecíficos (Stebbins 1971), situación que sería reportada por primera vez para el género.

Desde el punto de vista citológico, en esta sección (sensu Cabrera 1937) sólo se conoce el cariotipo de C. chilensis (Baeza \& Schrader 2005). Por lo que el objetivo de este trabajo es describir el cariotipo de C. elegans y C.x serrata, para posteriormente compararlos con el cariotipo de una nueva población de $C$. chilensis.

\section{MATERIALES Y MÉTODOS}

Se estudió una población de Chaetanthera chilensis (ca. 200 individuos) recolectada en Chile, Región del Biobío, 
Provincia de Nuble, espinal de Acacia caven, entre Chillán y entrada a Bulnes, $80 \mathrm{~m}\left(31^{\circ} 41^{\prime} \mathrm{S} / 72^{\circ} 15^{\prime} \mathrm{W}\right)$. 18-XII-2010, C. Baeza 4339; una población de Chaetanthera elegans (ca. 50 individuos), recolectada en la Región del Biobío, Provincia de Biobío, Parque Nacional Laguna del Laja, meseta de los

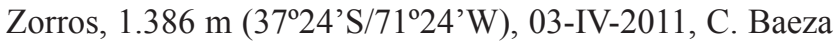
4348 y una población de Chaetanthera x serrata (ca. 30 individuos), recolectada en la Región del Biobío, Provincia de Concepción, Hualpén, predio de la Universidad de Concepción, $60 \mathrm{~m}$ (36 $\left.47^{\circ} \mathrm{S} / 73^{\circ} 10^{\prime} \mathrm{W}\right)$. 15-III-2011, C. Baeza 4345. El material de referencia está depositado en el Herbario de la Universidad de Concepción (CONC).

La metodología seguida es la indicada por Baeza et al. (2001, 2004). Raíces (1-2 cm de longitud) obtenidas a partir de plántulas fueron cortadas y pretratadas con una solución de 8 -hidroxiquinolina $(2 \mathrm{mM})$ durante $24 \mathrm{~h} \mathrm{a} 4{ }^{\circ} \mathrm{C}$. Posteriormente, fueron fijadas en una mezcla fresca de etanol / ácido acético (3:1) por $24 \mathrm{~h}$ y luego almacenadas en alcohol $70 \%$ $\mathrm{a}-20{ }^{\circ} \mathrm{C}$. Luego, las raíces fueron lavadas 3 veces en agua destilada durante 30 min y posteriormente tratadas con $\mathrm{HCl}$ $0,5 \mathrm{~N}$ durante $20 \mathrm{~min}$ a $42^{\circ} \mathrm{C}$. Posteriormente, se lavaron las raíces con agua destilada en 3 oportunidades. Por último, se realizó un aplastado (squash) del ápice de la raíz con orceína acética al $1 \%$ y se observó al microscopio. Se estudiaron 20 placas metafásicas en cada una de las poblaciones ( 2 placas por individuo, 10 individuos por población) y para cada población analizada se determinó el índice de asimetría del cariotipo (AsK \%) usando la fórmula descrita por Arano \& Saito (1980), el índice de asimetría de Huziwara (TF\%, 1962) y el índice de asimetría de Venora et al. (Syi, 2002). Los cromosomas se midieron con la ayuda del programa computacional "MicroMeasure 3.3" (Reeves 2001) y se clasificaron de acuerdo a los radios de los brazos $(\mathrm{R}=$ brazo largo / brazo corto) modificado de Levan et al. (1964).

\section{RESULTADOS}

En la Tabla I se resumen los datos obtenidos en las especies analizadas. Se puede observar que tanto $C$. elegans y $C$. x serrata presentan la misma fórmula cariotípica y comparten valores muy similares en relación a $\mathrm{R}$, AsK \%, TF\% y Syi. Sin embargo, la longitud total del cariotipo (LTC) de $C$. elegans es mayor que en $C$. x serrata. Ambas especies presentan el mismo cariotipo, con cromosomas en su mayoría metacéntricos, a excepción de los pares 5 y 11 que son submetacéntricos (Fig. 1 B-C). En cambio, C. chilensis presenta valores diferentes a las otras dos especies y sólo el par cromosómico 8 es submetacéntrico y el resto de los cromosomas son metacéntricos (Fig. 1 A).

\section{A

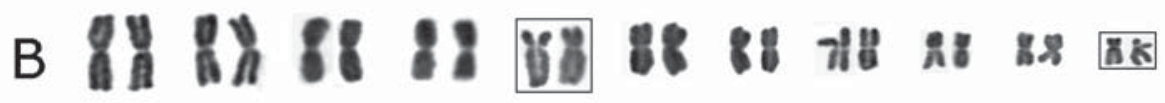 c}

Figura 1. Cariotipos de A. Chaetanthera chilensis, B. C. elegans y C. C. $x$ serrata. Escala $=5 \mu \mathrm{m}$.

Figure 1. Karyotypes of A. Chaetanthera chilensis, B. C. elegans and C. C. x serrata. Scale $=5 \mu \mathrm{m}$.

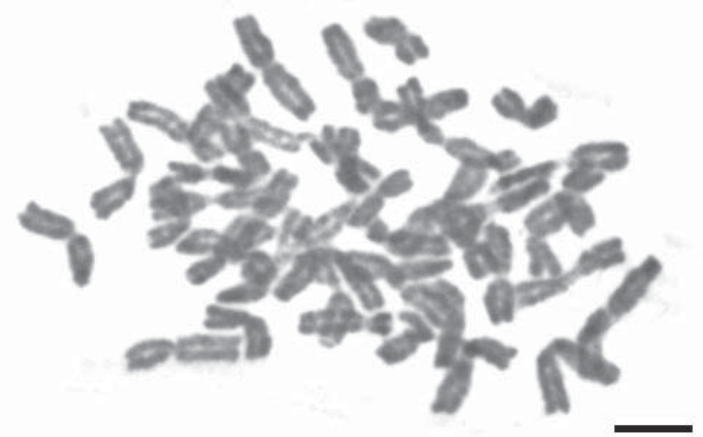

FIgURA 2. Placa metafásica tetraploide de Chaetanthera elegans. Escala $=5 \mu \mathrm{m}$.

Figure 2. Metaphasic tetraploid chromosomes of Chaetanthera elegans. Scale $=5 \mu \mathrm{m}$. 
TABLa I. Características del cariotipo de Chaetanthera chilensis, C. x serrata y C. elegans. LTC: longitud total de los cromosomas ( $\mu$ m); AsK \% = índice de asimetría de Arano y Saito (1980), TF\% = índice de asimetría de Huziwara (1962), Syi = índice de asimetría de Venora et al. (2002); R: cociente entre el par de cromosomas más largo y el más corto.

TABLE I. Comparison of karyotype characteristics of Chaetanthera chilensis, C. x serrata and C. elegans. TCL: total chromosome length $(\mu \mathrm{m}) ;$ AsK $\%=$ Asymmetry index of Arano and Saito (1980), TF\% = Asymmetry index of Huziwara (1962), Syi = Asymmetry index of Venora et al. (2002); R: ratio of the longest pair/shortest pair.

\begin{tabular}{lccc}
\hline CARACTERísticAS DEL CARIOTIPO & C. chilensis & C. $x$ serrata & C. elegans \\
\hline 2n & 22 & 22 & 22 \\
Fórmula cariotípica & $10 \mathrm{~m}+1 \mathrm{sm}$ & $9 \mathrm{~m}+2 \mathrm{sm}$ & $9 \mathrm{~m}+2 \mathrm{sm}$ \\
LTC $(\mu \mathrm{m})$ & $135,6 \pm 2,4$ & $139,6 \pm 2,9$ & $146,2 \pm 5,2$ \\
$\mathrm{R}$ & 1,7 & 2,2 & 2,1 \\
AsK $\%$ & 53,98 & 54,60 & 54,53 \\
TF\% & 46,02 & 45,4 & 45,47 \\
Syi & 85,23 & 83,20 & 83,4 \\
\hline
\end{tabular}

\section{DISCUSIÓN}

Baeza \& Schrader (2005) realizaron un análisis citogenético utilizando FISH en una población de C. chilensis proveniente de Quillón, en la Región del Biobío (Chile) y los valores relacionados con los índices de asimetría y LTC encontrados son muy similares a esta nueva población analizada. Ello muestra la estabilidad cariológica de esta especie, que presenta una amplia distribución en Chile y bastante variabilidad morfológica, pero no a nivel de su cariotipo. Chaetanthera serrata, sensu Cabrera (1937), es una especie presente en las provincias australes de Chile y aparece muy relacionada con C. chilensis y C. elegans (Hershkovitz et $a l$. 2006). Davies (2010) concluye en su revisión del género Chaetanthera en Chile que esta especie es un híbrido fértil entre $C$. chilensis y $C$. elegans. Morfológicamente, $C . \mathrm{x}$ serrata es muy similar a $C$. chilensis y la presencia de rizomas horizontales largos y hojas muy estrechas es el carácter que permite separarla. Difiere morfológicamente de C. elegans, ya que esta última presenta una estatura mayor y los capítulos son más grandes, además de no presentar rizomas horizontales. Un punto muy interesante que se destaca en el trabajo de Davies (2010) es la presencia en Chile de una zona de alto grado de endemismo y biodiversidad donde existe hibridización en Chaetanthera, ubicado entre laguna del Maule y Chillán, justo el área que comparten estas tres especies. Según esta autora, en esta área están ocurriendo procesos de hibridación que complican la determinación de ejemplares que crecen en este lugar. Por otro lado, esta autora señala que los híbridos producen pocos frutos viables y muy baja dispersión. Esta situación es corroborada en esta investigación, puesto que el porcentaje de germinación de C. x serrata no superó el $10 \%$, en cambio en C. chilensis y C. elegans la germinación fue prácticamente de un $100 \%$.
El probable híbrido C. x serrata comparte el mismo cariotipo y los mismos índices de asimetría con C. elegans, por lo que correspondería a un híbrido homoploide que mantiene el número cromosómico de ambos progenitores, pero las características cariológicas de sólo uno de ellos. Esta situación es poco usual en los procesos de hibridación, normalmente los híbridos interespecíficos constituyen líneas alopoliploides. Las especies híbridas homoploides pueden llegar a quedar aisladas por selección ecológica que favorece la formación de líneas híbridas, más que por diferencias en el número cromosómico con sus padres, como es el caso de los alopoliploides (Gross \& Rieseberg 2005, Smith et al. 2008). Esto es lo que podría estar ocurriendo en $C$. $x$ serrata dado que ocupa ambientes diferentes a las especies de las cuales se habría originado. C. chilensis se distribuye ampliamente entre Valparaíso y Concepción en ambas cordilleras y en el Valle Central. C. elegans crece principalmente en la Cordillera de los Andes y de la Costa, en las Regiones del Biobío y la Araucanía y C. x serrata se encuentra principalmente en las partes bajas de la Cordillera de la Costa y en el Valle Central entre Concepción y Temuco (Davies 2010).

Si bien la existencia de híbridos homoploides se conoce desde hace varias décadas (Randolph et al. 1961, Randolph 1966), últimamente, han aumentado los estudios sobre este mecanismo de especiación (Arnold 1993, Buerkle et al. 2000, Smith et al. 2008, Masuelli et al. 2009).

Eventualmente, si $C$. x serrata no fuera un híbrido, siguiendo el criterio de Cabrera (1937) y Hershkovitz et al. (2006), esta especie estaría muy emparentada con $C$. chilensis y C. elegans, ya que comparten el mismo número diploide de cromosomas y prácticamente el mismo cariotipo, situación que también ha sido encontrada en otros géneros de Asteraceae, como es el caso de Haplopappus (Baeza \& 
Schrader 2005) e Hypochaeris (Weiss-Schneeweiss et al. 2003, Baeza et al. 2007). Finalmente, es importante destacar que se observó la presencia de tetraploides en C. elegans (Fig. 2). La frecuencia de tetraploides fue muy baja ya que se encontró solo en un individuo. Este es el primer reporte para esta especie de Chaetanthera.

\section{AGRADECIMIENTOS}

Se agradece al Departamento de Botánica y a la Dirección de Investigación de la Universidad de Concepción por las facilidades otorgadas.

\section{BIBLIOGRAFÍA}

Arano, H. \& H. Saito. 1980. Cytological studies in family Umbelliferae 5. Karyotypes of seven species in subtribe Seselinae. La Kromosomo 2(17): 471-480.

ArNold, M. 1993. Iris nelsonii (Iridaceae): origin and genetic composition of a homoploid hybrid species. American Journal of Botany 80: 577-583.

Baeza, C., G. Kottirsch, J. Espejo \& R. Reinoso. 2001. Recuentos cromosómicos en plantas chilenas. I. Gayana Botánica 58: $133-137$

Baeza, C. \& O. Schrader. 2005. Karyotype analysis in Chaetanthera chilensis (Willd.) DC. and Chaetanthera ciliata Ruiz et Pavón (Asteraceae) by double fluorescence in situ hybridization. Caryologia 58(4): 332-338.

Baeza, C., E. Vosyka \& T. Stuessy. 2004. Recuentos cromosómicos en plantas que crecen en Chile. II. Darwiniana 42: 25-29.

Baeza, M., C. Cabezas, A. Terrab, T. Stuessy, E. Ruiz \& M. Negritto. 2007. Estudios cromosómicos en especies de Hypochaeris L. (Asteraceae, Lactuceae) de Chile. Gayana Botánica 64(2): 244-248.

Buerkle, C.A., R. J. Morrisì, M.A. Asmussen \& L.H. Rieseberg. 2000. The likelihood of homoploid hybrid speciation. Heredity $84: 441-451$.

CABRERA,A. 1937. Revisión del género Chaetanthera (Compositae). Revista del Museo de La Plata, Sección Botánica 1: 87215 .
DAvies, A. 2010. A systematic revision of Chaetanthera Ruiz \& Pav., and the reinstatement of Oriastrum Poepp. \& Endl. (Asteraceae: Mutisieae). Dissertation der Fakultaet fuer Biologie der Ludwig-Maximilians-Universitaet Muenchen. $316 \mathrm{pp}$.

Gross, B.L \& L.H. RieseberG. 2005. The ecological genetics of homoploid hybrid speciation. Journal of Heredity 96(3): 241-252.

Hershkovitz, M., M.T.K. Arroyo, C. Bell \& L. Hinojosa. 2006. Phylogeny of Chaetanthera (Asteraceae: Mutisieae) reveals both ancient and recent origins of the high elevation lineages. Molecular Phylogenetics and Evolution 41: 594605.

Huziwara, Y. 1962. Karyotype analysis in some genera of Compositae. VIII. Further studies on the chromosomes of Aster. American Journal of Botany 49: 116-119.

Levan, A., K. Fredga \& A. Sandberg. 1964. Nomenclature for centromeric position on chromosomes. Hereditas 52: 201220.

Masuelli, R.W., E. Camadro, L.E Erazzu, M. Bedogni \& C. MARFIL. 2009. Homoploid hybridization in the origin and evolution of wild diploid potato species. Plant Systematics and Evolution 277:143-151.

RANDOLPH, L. 1966. Iris nelsonii, a new species of Louisiana Iris of hybrid origin. Baileya 14: 143-169.

Randolph, L., J. Mitra \& I. Nelson. 1961. Cytotaxonomic studies of Louisiana Irises. Botanical Gazette 123: 125-133.

ReEves, A. 2001. MicroMeasure: a new computer program for the collection and analysis of cytogenetic data. Genome 44: 239-443.

Smith S.D., V.J. Kolberg \& D.A. Baum. 2008. Morphological and cytological evidence for homoploid hybridization in Ichroma (Solanaceae). Madroño 55(4): 280-284.

StebBins, G. 1971. Chromosomal evolution in the higher plants. Edward Arnold Publishing, London. 216 pp.

Venora, G., S. Blangiforti, M. Ruffini Castiglioni, D. Pignone, F. Losavio \& R. CREmoninI. 2002. Chromatin organisation and computer arded karyotyping of Triticum durum Desf. cv Timilia. Caryologia 55: 91-98.

Weiss-Schneeweiss, H., T. Stuessy, S. Siljak-Yakovlev, C. Baeza \& J. PARKer. 2003. Karyotype evolution in South American species of Hypochaeris (Asteraceae, Lactuceae). Plant Systematics and Evolution 241: 171-184.

Recibido: 14.12.11

Aceptado: 25.07.12 